\title{
The clinicopathological features of patients with membranous nephropathy
}

This article was published in the following Dove Press journal: International Journal of Nephrology and Renovascular Disease

\author{
Hanyu Zhu',* \\ Qiuxia $\operatorname{Han}^{2, *}$ \\ Dong Zhang' \\ Yong Wang' \\ Jing $\mathrm{GaO}^{3}$ \\ Xiaoli Yang' \\ Wenjia Geng ${ }^{4}$ \\ Xiangmei Chen' \\ 'Department of Nephrology, Chinese \\ PLA General Hospital, Chinese PLA \\ Institute of Nephrology, State Key \\ Laboratory of Kidney Diseases, \\ National Clinical Research Center \\ of Kidney Diseases, Beijing Key \\ Laboratory of Kidney Disease, \\ Beijing, China; ${ }^{2}$ Department of \\ Nephrology, The First Affiliated \\ Hospital of Zhengzhou University, \\ Zhengzhou, China; ${ }^{3}$ Department \\ of Clinical Biochemistry, Chinese \\ PLA General Hospital, Beijing, \\ China; ${ }^{4}$ Department of Nephrology, \\ Guangdong Provincial Hospital of \\ Chinese Medicine, Guangzhou, China \\ * These authors contributed equally \\ to this work
}

Correspondence: Dong Zhang; Yong Wang

Department of Nephrology, Chinese PLA General Hospital, Chinese PLA Institute of Nephrology, State Key Laboratory of Kidney Diseases, National Clinical Research Center for Kidney Diseases, Beijing Key Laboratory of Kidney Disease, 28 Fuxing Road, Haidian District, Beijing, 100853, China Email Dongzhang30I30I@I26.com; Wangyong30I30I@aliyun.com
Background: Membranous nephropathy (MN) represents a distinct glomerular disease which has been considered as a major cause of nephrotic syndrome (NS) in adults. Evidences show that the clinicopathological features of MN are various among MN cases. This study aimed to summarize and analyze the clinicopathological features of patients with $\mathrm{MN}$.

Methods: A total of $231 \mathrm{MN}$ patients were recruited in this study. Their clinical and pathological features were collected and analyzed according to their age, gender, pathological stages, and anti-phospholipase A2 receptor (anti-PLA2R) antibodies tests.

Results: Among the $231 \mathrm{MN}$ cases, the ratio of male to female was 1.47 and the mean age was $47.43 \pm 14.32$ years. Altogether, $163(70.6 \%)$ cases were positive for NS. Their serum antiPLA2R, body mass index, total cholesterol, triglyceride, low density lipoprotein cholesterol, D2, IgA, and IgE were increased, but IgG was decreased. The majority of the patients were middle aged and old aged. In addition, the pathological stage was significantly correlated with gender $(P=0.038)$, creatinine, $(P=0.021)$ and $\operatorname{IgE}(P=0.003)$. A total of $74.9 \% \mathrm{MN}$ patients were found to be positive for anti-PLA2R antibodies, and they were more likely to have abnormal serum indices.

Conclusion: The major clinicopathological characteristics of $\mathrm{MN}$ patients are summarized in this study. Male and elder MN cases are likely to have rapid disease progression. Advanced pathological stages and being positive for anti-PLA2R antibodies may be potential indicators for disease activity of MN.

Keywords: pathological features, clinical manifestation, membranous nephropathy

\section{Introduction}

Membranous nephropathy $(\mathrm{MN})$ represents a distinct glomerular disease. ${ }^{1}$ It has been considered as one of the most frequent types of nephrotic syndrome (NS) among adults worldwide, accounting for approximately $25 \%-40 \%$ of all the adults with NS., ${ }^{2,3} \mathrm{MN}$ can be classified into primary $\mathrm{MN}$, also known as idiopathic $\mathrm{MN}$ (iMN), and secondary MN (sMN) based on different pathogenesis, and the most common subtype is iMN, accounting for approximately $75 \%$ of $\mathrm{MN}$ cases. ${ }^{4} \mathrm{sMN}$ could be induced by various conditions, such as malignancies (breast, ovarian, lung, and stomach cancers, and lymphoproliferative disorders), infections (human immunodeficiency virus, malaria, hepatitis B and C viruses), some systemic autoimmune diseases (rheumatoid arthritis and lupus), and the consumption of drugs and toxins. ${ }^{5,6} \mathrm{MN}$ is characterized by non-selective proteinuria, with the nephrotic range of more than $3.5 \mathrm{~g} / \mathrm{day}{ }^{7}$ Patients diagnosed with $\mathrm{MN}$ have a high risk of developing end-stage renal disease. Therefore, 
further understanding of the clinicopathological characteristics and disease progression is crucial for diagnosis and treatment of $\mathrm{MN}$.

According to the statistics, the clinical manifestation of $\mathrm{MN}$ is variable among $\mathrm{MN}$ cases, as well as in the disease prognosis. ${ }^{8}$ Data have revealed that $\mathrm{MN}$ is common in middle and old age groups with the peak age of 31-60 years. ${ }^{9}$ The incidence of $\mathrm{MN}$ is different between men and women, and the ratio of male to female is approximately $2: 1 .{ }^{10}$ In previous studies, some typical clinical manifestations have been identified in patients with $\mathrm{MN}$, such as NS, edema, hypertension, renal failure, and microscopic hematuria. ${ }^{11}$ According to the guidelines of pathology, $\mathrm{MN}$ cases can be divided into four pathological stages (stages I, II, III, and IV). ${ }^{12,13}$ Renal dysfunction can usually be detected in patients who have had $\mathrm{MN}$ for 5-10 years and pathological stage III or IV. ${ }^{14}$ Given the diverse clinicopathological characteristics of $\mathrm{MN}$ patients, a better understanding of these features is necessary for $\mathrm{MN}$ detection and treatment.

In the present study, we retrospectively analyzed the clinicopathological features of patients with $\mathrm{MN}$.

\section{Ethics statement}

The research of investigating clinical and laboratory data of in-hospital patients for "Analysis of clinicopathological features for patients with membranous nephropathy" was a retrospective study and has been evaluated; it was confirmed that the protocols were conducted in accordance with the Declaration of Helsinki and approved by the Ethics Committee of Chinese PLA General Hospital. Signed informed consents were obtained from all the participants and their families. All of the clinicopathological features and personal information were made anonymous in the current study.

\section{Methods}

\section{Patients}

A total of 231 patients who were diagnosed with $\mathrm{MN}$ at Chinese PLA General Hospital from January 1, 2013 to March 15, 2016 were enrolled in this study. The diagnoses were based on the pathological evaluation of percutaneous renal biopsy under B ultrasound guidance. Primary MN was identified without secondary causes based on clinical evaluations, such as lupus, chronic infection, tumors, and drug exposure. Moreover, the primary $\mathrm{MN}$ individuals were negative for antinuclear antibodies, anti-neutrophil cytoplasmic antibodies, and hepatitis virus tests. If the collected patients presented the following clinical symptoms, they were confirmed as having NS: heavy proteinuria: $\geq 50 \mathrm{mg} / \mathrm{kg} /$ day (or $\geq 40 \mathrm{mg} / \mathrm{m}^{2} / \mathrm{h}$ ), or a proteinuria/creatininuria ratio $>2(\mathrm{mg} / \mathrm{mg})$; serum $\mathrm{ALB}<25 \mathrm{~g} / \mathrm{L}$; edema. The medical history, clinical and pathological data of the patients were routinely recorded at biopsy. The pathological stage of the patients was also evaluated by two independent pathologists. In addition, the clinical characteristics of the collected patients were also recorded, including age, gender, blood pressure, urine sugar, and body mass index (BMI). We collected the basic characteristics of the patients at biopsy from the medical records. Signed informed consents were obtained from all the participants or their families.

\section{Data collection}

All of the patients underwent percutaneous renal biopsy with the guidance of ultrasound, and the collected renal samples were embedded in paraffin. The pathological stage of the renal samples was evaluated by two independent pathologists following the methods detailed by Churg and Ehrenreich. ${ }^{15}$ Nineteen clinical parameters were tested from the urine and serum specimens, including anti-phospholipase A2 receptor (anti-PLA2R) antibodies, ALT, AST, total protein (TP), ALB, total bilirubin (TB), direct bilirubin (DB), ALP, GGT, glucose (GLU), urea nitrogen (UN), creatinine $(\mathrm{Cr})$, uric acid (Ua), total cholesterol (TC), triglyceride (TG), $\mathrm{CK}, \mathrm{LDH}$, high density lipoprotein cholesterol (HDL), low density lipoprotein cholesterol (LDL), and D2. Moreover, immunofluorescence assay was carried out to detect the serum IgA, IgG, IgM, IgE, C3, and C4. For these analyses, Hitachi 7500 electron microscope was adopted for the electron microscopy observations.

\section{Statistical analysis}

All the data used were expressed as mean $\pm \mathrm{SD}$, and the statistical analyses were conducted with SPSS 18.0 software (SPSS Inc., Chicago, IL, USA). The comparison for continuous variables between two groups were carried out by Student's $t$-test, while chi-square test was used to analyze the categorical variables between two groups. $P<0.05$ was considered as statistically significant.

\section{Results}

\section{General data and clinical manifestation of the $\mathrm{MN}$ patients}

A total of $231 \mathrm{MN}$ patients including 219 with primary $\mathrm{MN}$ and 12 with sMN were included in our study. The sMN cases were all caused by viral hepatitis infection. There were 135 males and 96 females with the ratio of male to female being 1.47. The mean age of the patients was 
$47.43 \pm 14.32$ years (age range of 16-79 years). As shown in Figure 1, most of the patients were 41-59 years old, accounting for $45 \%$ of the patients. A total of 76 patients $(33 \%)$ were $\leq 40$ years, and 52 cases $(22 \%)$ were $\geq 60$ years. Among all these patients, $82(35.5 \%)$ cases had hypertension and $28(12.1 \%)$ had diabetes, while 163 (70.6\%) patients suffered from NS. The average results of systolic and diastolic blood pressure were $132.39 \pm 22.18$ and $81.85 \pm 12.21 \mathrm{mmHg}$, respectively (Table 1).

All the clinical parameters were summarized in Table 1. According to the analyses in renal tissues and blood samples, we found increased serum anti-PLA2R antibodies with a mean concentration of $95.45 \pm 165.37$, and $173(74.9 \%) \mathrm{MN}$ cases were positive to anti-PLA2R antibodies test. Compared to the normal reference range, the BMI, TC, TG, LDL, D2, $\operatorname{IgA}$, and $\operatorname{IgE}$ were increased, but the $\operatorname{IgG}$ was decreased in patients with $\mathrm{MN}$. Other clinical features were distributed within the normal range in all the patients with $\mathrm{MN}$.

\section{Effects of age on clinicopathological features of patients with $\mathrm{MN}$}

All the patients were classified into two groups based on their age: $\leq 50$ years group $(n=125)$ and $>50$ years group $(n=106)$.

The clinicopathological parameters were compared between the two groups. From the results detailed in Table 2, we found that hypertension, anti-PLA2R antibodies, AST, UN, CK, and IgA were significantly increased in $>50$ years group, while BMI, TP, ALB, TB, and Ua were significantly decreased in the $>50$ years group (all $P<0.05$ ). However, no difference was observed for the other clinical parameters between the two age groups (all $P>0.05$ ).

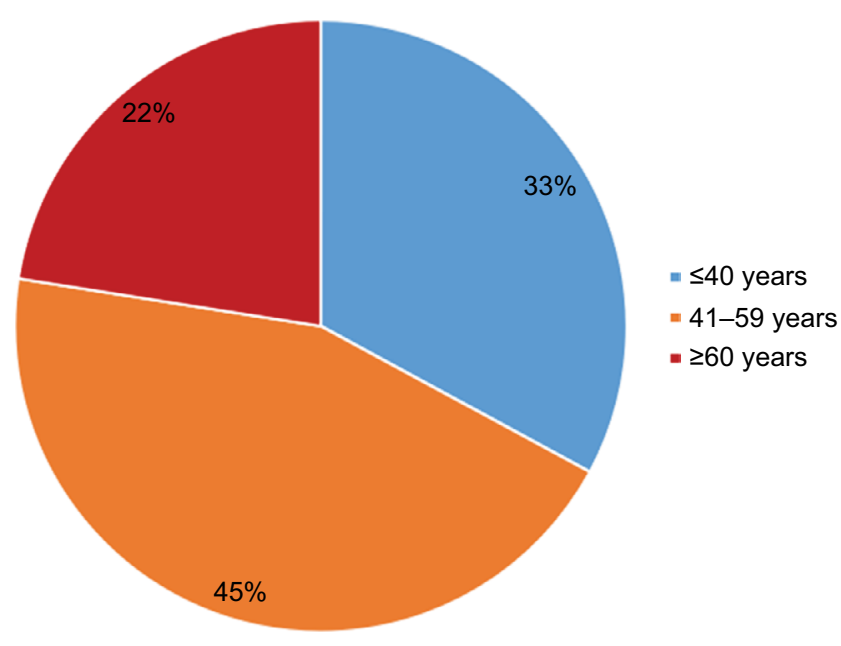

Figure I Membranous nephropathy patients stratified according to age. Note: The majority of the patients were middle and old aged.
Table I Summary of the clinicopathological features of MN patients

\begin{tabular}{|c|c|c|}
\hline Parameters & Results & $\begin{array}{l}\text { Reference } \\
\text { range }\end{array}$ \\
\hline Age (years) & $47.43 \pm 14.32$ & - \\
\hline Gender (male : female) & $135: 96$ & - \\
\hline Blood pressure (mmHg) & $|32.39 \pm 22.18 / 8| .85 \pm|2.2|$ & - \\
\hline NS & $163(70.6 \%)$ & - \\
\hline $\mathrm{BMI}\left(\mathrm{Kg} / \mathrm{m}^{2}\right)$ & $25.87 \pm 4.75$ & $18.5-24.9$ \\
\hline anti-PLA2R (RU/mL) & $95.45 \pm 165.37$ & - \\
\hline PLA2R positive (n, \%) & 173 (74.9\%) & - \\
\hline ALT (U/L) & $21.08 \pm 16.48$ & $0-40$ \\
\hline AST (U/L) & $18.60 \pm 9.29$ & $0-40$ \\
\hline $\mathrm{TP}(\mathrm{g} / \mathrm{L})$ & $49.44 \pm 10.02$ & $55-80$ \\
\hline ALB $(g / L)$ & $26.92 \pm 6.98$ & $35-50$ \\
\hline TB $(\mu \mathrm{mol} / \mathrm{L})$ & $7.64 \pm 3.52$ & $0-21$ \\
\hline $\mathrm{DB}(\mu \mathrm{mol} / \mathrm{L})$ & $1.46 \pm 1.14$ & $0-8.6$ \\
\hline ALP (U/L) & $59.14 \pm 16.68$ & $0-130$ \\
\hline GGT (U/L) & $34.94 \pm 48.22$ & $0-50$ \\
\hline GLU (mmol/L) & $4.95 \pm 1.24$ & $3.4-6.2$ \\
\hline UN (mmol/L) & $5.22 \pm 2.42$ & I.8-7.5 \\
\hline $\mathrm{Cr}(\mu \mathrm{mol} / \mathrm{L})$ & $75.10 \pm 24.66$ & $30-110$ \\
\hline $\mathrm{Ua}(\mu \mathrm{mol} / \mathrm{L})$ & $346.97 \pm 95.84$ & $104-444$ \\
\hline $\mathrm{TC}(\mathrm{mmol} / \mathrm{L})$ & $7.07 \pm 2.34$ & $3.1-5.7$ \\
\hline $\mathrm{TG}(\mathrm{mmol} / \mathrm{L})$ & $2.58 \pm 1.90$ & $0.4-1.7$ \\
\hline CK (U/L) & $102.65 \pm 139.16$ & $2-200$ \\
\hline LDH (U/L) & $193.60 \pm 57.98$ & $40-250$ \\
\hline $\mathrm{HDL}(\mathrm{mmol} / \mathrm{L})$ & $1.44 \pm 0.52$ & $1-1.6$ \\
\hline LDL (mmol/L) & $4.85 \pm 2.03$ & $0-3.4$ \\
\hline D2 ( $\mu g / L)$ & $1.26 \pm 2.28$ & $0.0-0.5$ \\
\hline $\lg \mathrm{A}(\mathrm{mg} / \mathrm{dL})$ & $215.41 \pm 84.18$ & $70-180$ \\
\hline $\lg G(\mathrm{mg} / \mathrm{dL})$ & $625.48 \pm 282.06$ & $700-1600$ \\
\hline $\operatorname{lgM}(\mathrm{mg} / \mathrm{dL})$ & $116.30 \pm 78.30$ & $40-230$ \\
\hline $\lg \mathrm{E}(\mathrm{mg} / \mathrm{dL})$ & $|26.13 \pm| 88.48$ & $0-100$ \\
\hline C3 (mg/dL) & $114.86 \pm 24.32$ & $90-180$ \\
\hline $\mathrm{C} 4$ (mg/dL) & $28.19 \pm 9.83$ & $10-40$ \\
\hline Diabetes & $28(12.1 \%)$ & - \\
\hline Viral hepatitis & $12(5.2 \%)$ & - \\
\hline Hepatitis B & $10(4.3 \%)$ & - \\
\hline Hepatitis C & $2(0.9 \%)$ & - \\
\hline Pathological stage (III) & $10(4.3 \%)$ & - \\
\hline
\end{tabular}

Notes: - , no data. Data are presented as mean \pm SD and $n$ (\%). Bold data is statistically significant.

Abbreviations: BMI, body mass index; TC, total cholesterol; $\mathrm{Cr}$, creatinine; DB, direct bilirubin; GLU, glucose; HDL, high density lipoprotein cholesterol; LDL, low density lipoprotein cholesterol; MN, membranous nephropathy; NS, nephrotic syndrome; TB, total bilirubin; TG, triglyceride; TP, total protein; Ua, uric acid; UN, urea nitrogen.

\section{Clinicopathological characteristics according to gender}

To uncover the relationship between the gender of patients and their clinicopathological features, the patients were divided into male group and female group. From the analysis results listed in Table 3, we found that patients with more advanced pathological stage were male $(P=0.038)$. Besides, the ALT, DB, GGT, UN, Cr, Ua, CK, and IgE in males were 
Table 2 Clinicopathological features of patients stratified according to age

\begin{tabular}{|c|c|c|c|}
\hline \multirow[t]{2}{*}{ Parameters } & \multicolumn{2}{|l|}{ Age range } & \multirow[t]{2}{*}{$P$-value } \\
\hline & $\begin{array}{l}\leq 50 \text { years } \\
(n=\mid 25)\end{array}$ & $\begin{array}{l}>50 \text { years } \\
(n=106)\end{array}$ & \\
\hline Gender (male : female) & $78: 47$ & $57: 49$ & 0.185 \\
\hline Hypertension (\%) & 24.0 & 49.1 & 0.000 \\
\hline Diabetes (\%) & 9.6 & 15.1 & 0.202 \\
\hline Viral hepatitis (\%) & 5.6 & 4.7 & 0.763 \\
\hline NS (\%) & 65.6 & 76.4 & 0.072 \\
\hline $\mathrm{BMI}\left(\mathrm{Kg} / \mathrm{m}^{2}\right)$ & $26.05 \pm 5.29$ & $25.67 \pm 4.01$ & 0.010 \\
\hline Pathological stage (III) (\%) & 6.4 & 1.9 & 0.093 \\
\hline Anti-PLA2R (RU/mL) & $66.22 \pm 104.33$ & $128.80 \pm 211.13$ & 0.001 \\
\hline $\operatorname{ALT}(\mathrm{U} / \mathrm{L})$ & $21.79 \pm 15.16$ & $20.44 \pm 18.00$ & 0.806 \\
\hline AST (U/L) & $17.47 \pm 5.93$ & $19.98 \pm 11.98$ & 0.019 \\
\hline $\mathrm{TP}(\mathrm{g} / \mathrm{L})$ & $50.23 \pm 11.19$ & $48.54 \pm 8.35$ & 0.003 \\
\hline ALB $(g / L)$ & $27.68 \pm 7.78$ & $26.05 \pm 5.80$ & 0.001 \\
\hline TB $(\mu \mathrm{mol} / \mathrm{L})$ & $7.67 \pm 3.79$ & $7.62 \pm 3.17$ & 0.048 \\
\hline $\mathrm{DB}(\mu \mathrm{mol} / \mathrm{L})$ & $1.40 \pm 0.93$ & $1.54 \pm 1.35$ & 0.401 \\
\hline ALP (U/L) & $54.81 \pm 15.64$ & $64.34 \pm 16.40$ & 0.456 \\
\hline GGT (U/L) & $36.1 I \pm 43.24$ & $35.24 \pm 56.26$ & 0.884 \\
\hline GLU (mmol/L) & $4.87 \pm 1.31$ & $5.04 \pm 1.14$ & 0.554 \\
\hline UN (mmol/L) & $4.72 \pm 1.75$ & $5.83 \pm 2.92$ & 0.004 \\
\hline $\mathrm{Cr}(\mu \mathrm{mol} / \mathrm{L})$ & $72.64 \pm 20.32$ & $78.02 \pm 28.69$ & 0.074 \\
\hline $\mathrm{Ua}(\mu \mathrm{mol} / \mathrm{L})$ & $356.58 \pm 104.41$ & $337.34 \pm 85.24$ & 0.015 \\
\hline $\mathrm{TC}(\mathrm{mmol} / \mathrm{L})$ & $7.12 \pm 2.41$ & $7.04 \pm 2.28$ & 0.318 \\
\hline $\mathrm{TG}(\mathrm{mmol} / \mathrm{L})$ & $2.54 \pm 1.73$ & $2.65 \pm 2.09$ & 0.221 \\
\hline CK (U/L) & $93.17 \pm 62.05$ & $113.38 \pm 193.91$ & 0.047 \\
\hline LDH (U/L) & $190.93 \pm 62.96$ & $198.00 \pm 52.83$ & 0.228 \\
\hline $\mathrm{HDL}(\mathrm{mmol} / \mathrm{L})$ & $1.39 \pm 0.50$ & $1.50 \pm 0.54$ & 0.787 \\
\hline LDL (mmol/L) & $4.93 \pm 2.05$ & $4.77 \pm 2.0 \mathrm{I}$ & 0.566 \\
\hline D2 $(\mu g / L)$ & $1.11 \pm 2.23$ & $1.44 \pm 2.34$ & 0.437 \\
\hline $\lg \mathrm{A}(\mathrm{mg} / \mathrm{dL})$ & $204.71 \pm 79.76$ & $226.94 \pm 88.33$ & 0.015 \\
\hline $\lg G(m g / d L)$ & $616.03 \pm 303.52$ & $632.76 \pm 257.16$ & 0.297 \\
\hline $\operatorname{lgM}(\mathrm{mg} / \mathrm{dL})$ & II $2.79 \pm 67.28$ & | | $9.77 \pm 89.7 \mid$ & 0.118 \\
\hline $\lg \mathrm{E}(\mathrm{mg} / \mathrm{dL})$ & $126.67 \pm 197.85$ & $124.48 \pm 177.05$ & 0.702 \\
\hline C3 (mg/dL) & $114.64 \pm 25.13$ & $115.26 \pm 23.36$ & 0.090 \\
\hline $\mathrm{C} 4(\mathrm{mg} / \mathrm{dL})$ & $27.89 \pm 9.34$ & $28.49 \pm 10.39$ & 0.340 \\
\hline
\end{tabular}

Note: Data are presented as mean \pm SD unless otherwise stated. Bold data is statistically significant, $P<0.05$.

Abbreviations: BMI, body mass index; $\mathrm{TC}$, total cholesterol; $\mathrm{Cr}$, creatinine; $\mathrm{DB}$, direct bilirubin; GLU, glucose; HDL, high density lipoprotein cholesterol; LDL, low density lipoprotein cholesterol; NS, nephrotic syndrome; TB, total bilirubin; TG, triglyceride; TP, total protein; Ua, uric acid; UN, urea nitrogen.

remarkably higher than that in females $(P<0.05$ for all). Inversely, the results of anti-PLA2R antibodies and IgM were decreased in males compared with females (both $P<0.05$ ).

\section{Association of histological stage with clinicopathological characteristics of $\mathrm{MN}$ patients}

In this study, 221 (95.7\%) MN patients were pathologically diagnosed with stages I-II, and ten (4.3\%) cases were stage III. We also analyzed the clinical characteristics of MN patients according to their pathological stages. In Table 4,
Table 3 Clinicopathological features of patients stratified according to gender

\begin{tabular}{|c|c|c|c|}
\hline Parameters & $\begin{array}{l}\text { Male } \\
(n=135)\end{array}$ & $\begin{array}{l}\text { Female } \\
(n=96)\end{array}$ & $P$-value \\
\hline Age (years) & $47.04 \pm 14.70$ & $47.95 \pm 13.76$ & 0.280 \\
\hline Hypertension (\%) & 40.0 & 29.2 & 0.090 \\
\hline Diabetes (\%) & 11.9 & 12.5 & 0.882 \\
\hline Viral hepatitis (\%) & 3.7 & 7.3 & 0.226 \\
\hline NS (\%) & 74.1 & 65.6 & 0.165 \\
\hline BMI $\left(\mathrm{Kg} / \mathrm{m}^{2}\right)$ & $26.12 \pm 4.35$ & $25.54 \pm 5.24$ & 0.357 \\
\hline Pathological stage (III) (\%) & 6.7 & 1.0 & 0.038 \\
\hline Anti-PLA2R (RU/mL) & $77.83 \pm 119.78$ & $119.55 \pm 211.97$ & 0.002 \\
\hline ALT (U/L) & $24.21 \pm 19.60$ & $16.89 \pm 9.24$ & 0.016 \\
\hline AST (U/L) & $19.48 \pm 10.88$ & $17.42 \pm 6.23$ & 0.148 \\
\hline $\mathrm{TP}(\mathrm{g} / \mathrm{L})$ & $47.99 \pm 10.14$ & $51.5 I \pm 9.48$ & 0.863 \\
\hline $\operatorname{ALB}(g / L)$ & $26.37 \pm 7.26$ & $27.73 \pm 6.50$ & 0.271 \\
\hline TB $(\mu \mathrm{mol} / \mathrm{L})$ & $7.93 \pm 3.66$ & $7.25 \pm 3.27$ & 0.109 \\
\hline $\mathrm{DB}(\mu \mathrm{mol} / \mathrm{L})$ & $1.61 \pm 1.33$ & $1.26 \pm 0.76$ & 0.021 \\
\hline ALP (U/L) & $58.85 \pm 15.72$ & $59.61 \pm 17.96$ & 0.614 \\
\hline GGT (U/L) & $45.22 \pm 61.42$ & $22.31 \pm 17.34$ & 0.000 \\
\hline GLU (mmol/L) & $5.00 \pm 1.19$ & $4.87 \pm 1.30$ & 0.662 \\
\hline UN (mmol/L) & $5.83 \pm 2.72$ & $4.38 \pm 1.60$ & 0.019 \\
\hline $\mathrm{Cr}(\mu \mathrm{mol} / \mathrm{L})$ & $84.73 \pm 24.40$ & $61.57 \pm 17.59$ & 0.014 \\
\hline $\mathrm{Ua}(\mu \mathrm{mol} / \mathrm{L})$ & $386.04 \pm 93.44$ & $293.91 \pm 71.80$ & 0.007 \\
\hline $\mathrm{TC}(\mathrm{mmol} / \mathrm{L})$ & $7.20 \pm 2.46$ & $6.93 \pm 2.19$ & 0.064 \\
\hline TG $(\mathrm{mmol} / \mathrm{L})$ & $2.7 I \pm 2.03$ & $2.43 \pm 1.7 \mid$ & 0.290 \\
\hline CK (U/L) & $121.93 \pm 171.80$ & $75.12 \pm 62.21$ & 0.019 \\
\hline LDH (U/L) & $196.90 \pm 64.39$ & $190.34 \pm 49.10$ & 0.209 \\
\hline $\mathrm{HDL}(\mathrm{mmol} / \mathrm{L})$ & $1.33 \pm 0.5 \mathrm{I}$ & $1.59 \pm 0.49$ & 0.408 \\
\hline LDL (mmol/L) & $4.98 \pm 2.14$ & $4.68 \pm 1.87$ & 0.089 \\
\hline D2 $(\mu \mathrm{g} / \mathrm{L})$ & $1.33 \pm 2.49$ & $1.17 \pm 1.96$ & 0.361 \\
\hline $\lg A(m g / d L)$ & $210.36 \pm 83.73$ & $221.20 \pm 85.23$ & 0.466 \\
\hline $\lg G(\mathrm{mg} / \mathrm{dL})$ & $583.46 \pm 288.10$ & $679.46 \pm 266.66$ & 0.428 \\
\hline $\operatorname{lgM}(\mathrm{mg} / \mathrm{dL})$ & $98.75 \pm 64.54$ & |39.88ะ88.99 & 0.022 \\
\hline $\operatorname{lgE}(\mathrm{mg} / \mathrm{dL})$ & $146.86 \pm 204.62$ & $96.30 \pm 159.14$ & 0.021 \\
\hline C3 (mg/dL) & || $4.82 \pm 22.9 \mid$ & $115.08 \pm 26.19$ & 0.294 \\
\hline $\mathrm{C} 4$ (mg/dL) & $28.95 \pm 10.01$ & $27.08 \pm 9.49$ & 0.513 \\
\hline
\end{tabular}

Note: Data are presented as mean \pm SD unless otherwise stated. Bold data is statistically significant, $P<0.05$.

Abbreviations: BMI, body mass index; TC, total cholesterol; $\mathrm{Cr}$, creatinine; DB, direct bilirubin; GLU, glucose; HDL, high density lipoprotein cholesterol; LDL, low density lipoprotein cholesterol; NS, nephrotic syndrome; TB, total bilirubin; TG, triglyceride; TP, total protein; Ua, uric acid; UN, urea nitrogen.

the results showed that the patients with more advanced pathological stages were more frequently male than female $(P=0.038)$, moreover, the $\mathrm{MN}$ patients with advanced stages were more likely to have increased $\mathrm{Cr}(P=0.021)$ and $\operatorname{IgE}$ $(P=0.003)$ levels compared with those with stages I-II. There was no significant correlation between pathological stage and other clinical parameters (all $P>0.05$ ).

\section{The comparison of clinicopathological characteristics of $\mathrm{MN}$ patients based on their anti-PLA2R antibodies test}

Based on anti-PLA2R antibodies test, $\mathrm{MN}$ patients were divided into PLA2R positive group (173, 74.89\%), or negative group 
Table 4 Association of pathological stage with clinicopathological data of MN patients

\begin{tabular}{|c|c|c|c|}
\hline \multirow[t]{2}{*}{ Parameters } & \multicolumn{2}{|c|}{ Pathological stage } & \multirow[t]{2}{*}{$P$-value } \\
\hline & $I-I \mid(n=22 I)$ & III $(n=10)$ & \\
\hline Age (years) & $47.57 \pm 14.17$ & $43.90 \pm 17.17$ & 0.705 \\
\hline Gender (male : female) & $126: 95$ & $9: 1$ & 0.038 \\
\hline Hypertension (\%) & 34.8 & 50.0 & 0.327 \\
\hline Diabetes (\%) & 12.7 & 0 & 0.230 \\
\hline Viral hepatitis (\%) & 5.4 & 0 & 0.449 \\
\hline NS (\%) & 70.6 & 70.0 & 0.968 \\
\hline BMI $\left(\mathrm{Kg} / \mathrm{m}^{2}\right)$ & $25.84 \pm 4.70$ & $26.63 \pm 5.92$ & 0.364 \\
\hline anti-PLA2R (RU/mL) & $96.66 \pm 165.96$ & $59.83 \pm 148.42$ & 0.668 \\
\hline ALT (U/L) & $21.05 \pm 16.66$ & $23.80 \pm 12.89$ & 0.909 \\
\hline AST (U/L) & $18.70 \pm 9.36$ & $17.06 \pm 7.25$ & 0.856 \\
\hline $\mathrm{TP}(\mathrm{g} / \mathrm{L})$ & $49.19 \pm 9.84$ & $55.30 \pm 12.14$ & 0.548 \\
\hline ALB $(g / L)$ & $26.73 \pm 6.91$ & $31.35 \pm 7.15$ & 0.905 \\
\hline TB $(\mu \mathrm{mol} / \mathrm{L})$ & $7.63 \pm 3.54$ & $7.95 \pm 3.12$ & 0.902 \\
\hline $\mathrm{DB}(\mu \mathrm{mol} / \mathrm{L})$ & $1.45 \pm 1.15$ & $1.67 \pm 0.84$ & 0.675 \\
\hline ALP (U/L) & $59.34 \pm|6.7|$ & $55.14 \pm 15.48$ & 0.535 \\
\hline GGT (U/L) & $35.69 \pm 50.50$ & $36.34 \pm|9.3|$ & 0.508 \\
\hline GLU (mmol/L) & $4.96 \pm 1.25$ & $4.52 \pm 0.42$ & 0.163 \\
\hline UN (mmol/L) & $5.16 \pm 2.35$ & $6.58 \pm 3.54$ & 0.214 \\
\hline $\mathrm{Cr}(\mu \mathrm{mol} / \mathrm{L})$ & $74.50 \pm 23.86$ & $88.65 \pm 36.66$ & 0.021 \\
\hline $\mathrm{Ua}(\mu \mathrm{mol} / \mathrm{L})$ & $345.68 \pm 96.45$ & $393.5 I \pm 86.54$ & 0.197 \\
\hline TC $(\mathrm{mmol} / \mathrm{L})$ & $7.10 \pm 2.35$ & $6.5 \mathrm{I} \pm 2.33$ & 0.664 \\
\hline TG (mmol/L) & $2.59 \pm 1.93$ & $2.55 \pm 1.10$ & 0.502 \\
\hline CK (U/L) & $103.35 \pm 141.29$ & $80.06 \pm 56.64$ & 0.572 \\
\hline LDH (U/L) & $194.00 \pm 58.62$ & $198.38 \pm 58.63$ & 0.526 \\
\hline $\mathrm{HDL}(\mathrm{mmol} / \mathrm{L})$ & $\mathrm{I} .45 \pm 0.5 \mathrm{I}$ & $1.25 \pm 0.72$ & 0.717 \\
\hline LDL (mmol/L) & $4.87 \pm 2.04$ & $4.44 \pm 1.77$ & 0.483 \\
\hline $\mathrm{D} 2(\mu \mathrm{g} / \mathrm{L})$ & $1.30 \pm 2.32$ & $0.45 \pm 0.25$ & 0.136 \\
\hline $\lg \mathrm{A}(\mathrm{mg} / \mathrm{dL})$ & $216.08 \pm 84.79$ & $189.18 \pm 72.97$ & 0.507 \\
\hline $\lg G(m g / d L)$ & $617.93 \pm 275.25$ & $750.10 \pm 4|3.8|$ & 0.483 \\
\hline $\lg M(m g / d L)$ & $|17.55 \pm 79.2|$ & $81.73 \pm 42.58$ & 0.226 \\
\hline $\operatorname{lgE}(\mathrm{mg} / \mathrm{dL})$ & $119.70 \pm 180.77$ & $256.20 \pm 293.05$ & 0.003 \\
\hline C3 (mg/dL) & $115.09 \pm 24.17$ & $111.22 \pm 27.82$ & 0.552 \\
\hline $\mathrm{C} 4$ (mg/dL) & $28.35 \pm 9.89$ & $24.14 \pm 7.47$ & 0.301 \\
\hline
\end{tabular}

Note: Data are presented as mean \pm SD unless otherwise stated. Bold data is statistically significant, $P<0.05$.

Abbreviations: $\mathrm{BMI}$, body mass index; $\mathrm{TC}$, total cholesterol; $\mathrm{Cr}$, creatinine; $\mathrm{DB}$, direct bilirubin; GLU, glucose; HDL, high density lipoprotein cholesterol; LDL, low density lipoprotein cholesterol; MN, membranous nephropathy; NS, nephrotic syndrome; TB, total bilirubin; TG, triglyceride; TP, total protein; Ua, uric acid; UN, urea nitrogen.

(58, 25.11\%). Compared to negative group, there were more male patients in positive group $(P=0.034)$, moreover, they were more likely to present NS $(P<0.001)$. Besides, MN patients positive for anti-PLA2R antibodies showed significantly decreased TP $(P<0.001)$, ALB $(P<0.001)$, TB $(P=0.005)$, DB $(P=0.001), \operatorname{IgG}(P<0.001)$, and upregulated $\mathrm{CH}(P=0.003)$, CK $(P=0.020)$, LDL $(P=0.003)$, and D2 $(P=0.032)$ (Table 5).

\section{Discussion}

$\mathrm{MN}$ is a common disease of the urinary system, which is characterized by the accumulation of immune deposits on
Table 5 Comparison of clinicopathological data of MN patients based on their anti-PLA2R test

\begin{tabular}{|c|c|c|c|}
\hline \multirow[t]{2}{*}{ Parameters } & \multicolumn{2}{|l|}{ Anti-PLA2R } & \multirow[t]{2}{*}{$P$-value } \\
\hline & $\begin{array}{l}\text { Positive } \\
(n=\mid 73)\end{array}$ & $\begin{array}{l}\text { Negative } \\
(n=58)\end{array}$ & \\
\hline Age (years) & $47.37 \pm 14.35$ & $47.55 \pm 14.24$ & 0.933 \\
\hline Gender (male : female) & $108: 65$ & $27: 31$ & 0.034 \\
\hline Hypertension (\%) & 67 (38.73) & $15(25.86)$ & 0.076 \\
\hline Diabetes (\%) & $18(10.40)$ & $10(17.24)$ & 0.167 \\
\hline Viral hepatitis (\%) & II (6.3.6) & $\mathrm{I}(\mathrm{I} .72)$ & $0.30 \mathrm{I}^{\mathrm{a}}$ \\
\hline NS (\%) & I $33(76.88)$ & $30(51.72)$ & $<0.001$ \\
\hline BMI $\left(\mathrm{Kg} / \mathrm{m}^{2}\right)$ & $25.93 \pm 4.89$ & $25.71 \pm 4.30$ & 0.759 \\
\hline $\operatorname{ALT}(\mathrm{U} / \mathrm{L})$ & $20.36 \pm 16.17$ & $23.60 \pm 17.35$ & 0.196 \\
\hline AST (U/L) & $18.97 \pm 10.04$ & $17.60 \pm 6.45$ & 0.334 \\
\hline $\mathrm{TP}(\mathrm{g} / \mathrm{L})$ & $47.66 \pm 9.63$ & $54.78 \pm 9.21$ & $<0.001$ \\
\hline ALB $(g / L)$ & $25.67 \pm 6.66$ & $30.70 \pm 6.54$ & $<0.001$ \\
\hline TB $(\mu \mathrm{mol} / \mathrm{L})$ & $7.27 \pm 3.10$ & $8.75 \pm 4.36$ & 0.005 \\
\hline $\mathrm{DB}(\mu \mathrm{mol} / \mathrm{L})$ & $1.31 \pm 0.79$ & $1.91 \pm 1.75$ & 0.001 \\
\hline ALP (U/L) & $58.63 \pm 15.54$ & $60.75 \pm 19.64$ & 0.403 \\
\hline GGT (U/L) & $35.86 \pm 53.07$ & $35.29 \pm 37.55$ & 0.941 \\
\hline GLU (mmol/L) & $4.93 \pm 1.26$ & $5.00 \pm 1.18$ & 0.705 \\
\hline UN (mmol/L) & $5.30 \pm 2.33$ & $5.02 \pm 2.68$ & 0.450 \\
\hline $\mathrm{Cr}(\mu \mathrm{mol} / \mathrm{L})$ & $75.58 \pm 22.72$ & $73.70 \pm 59.71$ & 0.615 \\
\hline $\mathrm{Ua}(\mu \mathrm{mol} / \mathrm{L})$ & $352.72 \pm 91.73$ & $332.92 \pm 108.54$ & 0.176 \\
\hline $\mathrm{TC}(\mathrm{mmol} / \mathrm{L})$ & $7.35 \pm 2.31$ & $6.28 \pm 2.29$ & 0.003 \\
\hline TG $(\mathrm{mmol} / \mathrm{L})$ & $2.70 \pm 2.03$ & $2.26 \pm 1.40$ & 0.125 \\
\hline CK (U/L) & $115.05 \pm 157.59$ & $65.60 \pm 38.08$ & 0.020 \\
\hline LDH (U/L) & $195.33 \pm 51.50$ & $190.79 \pm 75.82$ & 0.614 \\
\hline $\mathrm{HDL}(\mathrm{mmol} / \mathrm{L})$ & $1.42 \pm 0.50$ & $1.5 \mathrm{I} \pm 0.58$ & 0.277 \\
\hline LDL (mmol/L) & $5.08 \pm 2.03$ & $4.17 \pm 1.89$ & 0.003 \\
\hline D2 ( $\mu g / L)$ & $1.45 \pm 2.48$ & $0.70 \pm 1.39$ & 0.032 \\
\hline $\lg \mathrm{A}(\mathrm{mg} / \mathrm{dL})$ & $212.78 \pm 83.50$ & $221.30 \pm 97.29$ & 0.510 \\
\hline $\operatorname{lgG}(\mathrm{mg} / \mathrm{dL})$ & $578.53 \pm 268.00$ & $760.02 \pm 284.50$ & $<0.001$ \\
\hline $\lg M(\mathrm{mg} / \mathrm{dL})$ & 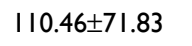 & | $32.67 \pm 93.85$ & 0.063 \\
\hline $\operatorname{lgE}(\mathrm{mg} / \mathrm{dL})$ & $|26.5| \pm 183.50$ & $123.12 \pm 203.40$ & 0.907 \\
\hline C3 (mg/dL) & $114.23 \pm 24.26$ & $117.02 \pm 24.44$ & 0.455 \\
\hline C4 (mg/dL) & $28.72 \pm 9.94$ & $26.48 \pm 9.34$ & 0.135 \\
\hline
\end{tabular}

Notes: a ${ }^{C}$ ase number less than 5 - corrected $P$-value was used. Data are presented as mean $\pm S D$ unless otherwise stated. Bold data is statistically significant, $P<0.05$. Abbreviations: BMI, body mass index; TC, total cholesterol; $\mathrm{Cr}$, creatinine; $\mathrm{DB}$, direct bilirubin; GLU, glucose; HDL, high density lipoprotein cholesterol; LDL, low density lipoprotein cholesterol; MN, membranous nephropathy; NS, nephrotic syndrome; TB, total bilirubin; TG, triglyceride; TP, total protein; Ua, uric acid; UN, urea nitrogen.

the outer surface of the glomerular basement membrane. ${ }^{16}$ Most MN cases are iMN, representing a major cause of idiopathic NS among the adult population. ${ }^{17}$ Despite various therapeutic strategies, the therapeutic effects are far from satisfactory. ${ }^{18-20}$ Most of iMN cases will suffer from NS, posing a serious threat to human health. ${ }^{21}$ And even worse, growing evidences have indicated that $\mathrm{MN}$ usually shows high rate of recurrence and long disease course, which may occur at any stage in life. ${ }^{22}$ So far, the molecular mechanisms underlying the progression of $\mathrm{MN}$ remain unclear, and the clinicopathological characteristics 
are variable in $\mathrm{MN}$ cases, bringing a heavy burden for $\mathrm{MN}$ diagnosis and treatment. ${ }^{23}$

In the current study, we investigated and analyzed the clinicopathological characteristics of 231 patients who were pathologically diagnosed with $\mathrm{MN}$. The ratio of male to female in the patients was 1.47 and the mean age was 47.43 \pm 14.32 . Most patients were 41-59 years old, accounting for $45 \%$ of all the cases. Among them, 163 (70.6\%) cases were positive for NS, which was in accordance with the data in the previous studies. In addition to these general data, the analysis of clinical parameters indicated that serum antiPLA2R antibodies, BMI, CH, TG, LDL, D2, IgA, and IgE were increased in the $\mathrm{MN}$ patients compared to the normal reference values, but IgG was decreased in MN patients. In subsequent analysis, we analyzed the clinical characteristics of MN patients according to their age. We found significant differences for hypertension, BMI, anti-PLA2R antibodies, AST, TP, ALB, TB, UN, Ua, CK, and IgA between MN patients in the $\leq 50$ years group and those in the $>50$ years group. MN patients with advanced age were more likely to exhibit poor performance in blood examinations, revealing the aggressive disease progression. The study carried out by Chen et al reported that old age and high blood indices were significantly correlated with poor prognosis of patients with iMN.$^{24}$ Therefore, timely and effective treatment and medical care is particularly important for older $\mathrm{MN}$ patients.

Gender has also been proven to be associated with disease progression in patients with MN. ${ }^{25}$ In our study, we investigated the clinical characteristics of $\mathrm{MN}$ patients based on their gender. The results showed that ALT, DB, GGT, UN, $\mathrm{Cr}$, Ua, CK, and IgE were remarkably higher in males than in females. These results might reveal that male MN patients are more likely to undergo rapid disease progression than female patients. The results are consistent with previous studies. A related study performed among Japanese MN cases demonstrated that male $\mathrm{MN}$ cases were more likely to develop end-stage renal diseases, and have a poor prognosis. ${ }^{25}$ Furthermore, it has been reported that with aging, men presented obviously greater decrements in renal functions than women, moreover, they were more likely to suffer from progressive renal diseases, such as MN, IgA nephropathy, and polycystic kidney disease. The mechanisms underlying the phenomenon might be related to the cell death induced by androgens. ${ }^{26}$ Inversely, anti-PLA2R antibodies and IgM were decreased in males compared with females. The abnormal results might be attributed to the relatively small sample size, different geographic areas, as well as the detection methods. Thus, further studies will be required to confirm our results.
According to the pathological classification method, $\mathrm{MN}$ can be divided into stages I, II, III, and IV. The MN stage I cases have normal glomeruli, which can be observed by light microscopy. ${ }^{27}$ However, for MN patients with stage II-IV, the capillary wall thicken and the deposits are gradually incorporated into the glomerular basement membrane. ${ }^{28}$ Pathological stage classification is an effective way to estimate disease progression. In our study, we found that the pathological stage was significantly correlated with patients' gender, Cr, and IgE. Advanced pathological stage was more likely to be observed in males than in females. Moreover, MN patients with advanced stage usually had increased $\mathrm{Cr}$ and $\mathrm{IgE}$ compared with those with stages I-II. $\mathrm{IgE}$ is an important factor in allergic reactions. Studies have demonstrated that patients with renal diseases showed high serum levels of IgE, moreover, serum IgE level might be significantly correlated with disease activity and clinical outcomes of NS. ${ }^{29,30}$ However, the functional roles of $\operatorname{IgE}$ in the pathogenesis of renal disease remains poorly understood. Further investigations are urgently needed to address the issues.

Anti-PLA2R antibodies is an important factor in the occurrence of MN. ${ }^{31}$ In general, anti-PLA2R antibodies is considered as a useful biomarker for early diagnosis and prognosis in patients with MN. ${ }^{32}$ In this study, we compared the baseline characteristics of $\mathrm{MN}$ patients according to their anti-PLA2R antibodies test results. The results demonstrated that $\mathrm{MN}$ cases who were positive for anti-PLA2R antibodies had significantly decreased TP, ALB, TB, DB, IgG; and upregulated $\mathrm{CH}, \mathrm{CK}, \mathrm{LDL}, \mathrm{D} 2$. Furthermore, they were more likely to present NS. The data revealed that patients in the anti-PLA2R antibodies positive group had rapid disease progression. The conclusion is in line with the previous studies. ${ }^{33}$ The podocyte lesion plays a key role in the etiology of MN. PLA2R is located at the surface of podocytes, and its abnormal expression may reveal the abnormal formation of immune complexes in situ, thus leading to damage in podocytes. ${ }^{34}$

\section{Conclusion}

In conclusion, the clinicopathological characteristics are diverse among $\mathrm{MN}$ patients. Male and elderly MN patients are more likely to undergo a rapid disease progression. Furthermore, the clinical characteristics are significantly correlated with pathological stages and anti-PLA2R antibodies level. Due to the relatively small sample size, the results obtained in our study should be verified by well-designed studies with large sample size. 


\section{Acknowledgments}

This study was supported by: 1) National key R\&D Program of China (2016YFC1305500); 2) the National Natural Science Foundation of China (nos. 61471399 and 61671479); 3) Innovation Nursery Fund of PLA General Hospital (no. 15KMZ04); 4) the National Natural Science Foundation of China (81401719).

\section{Disclosure}

The authors report no conflicts of interest in this work.

\section{References}

1. Ronco P, Debiec H. Membranous nephropathy: A fairy tale for immunopathologists, nephrologists and patients. Mol Immunol. 2015;68(1):57-62.

2. Li LS, Liu ZH. Epidemiologic data of renal diseases from a single unit in China: analysis based on 13,519 renal biopsies. Kidney Int. 2004;66(3):920-923.

3. Ponticelli C, Passerini P. Can prognostic factors assist therapeutic decisions in idiopathic membranous nephropathy? J Nephrol. 2010;23(2):156-163.

4. Dai H, Zhang H, He Y. Diagnostic accuracy of PLA2R autoantibodies and glomerular staining for the differentiation of idiopathic and secondary membranous nephropathy: an updated meta-analysis. Sci Rep 2015;5:8803.

5. Ayalon R, Beck LH Jr. Membranous nephropathy: not just a disease for adults. Pediatr Nephrol. 2015;30(1):31-39.

6. Basford AW, Lewis J, Dwyer JP, Fogo AB. Membranous nephropathy with crescents. J Am Soc Nephrol. 2011;22(10):1804-1808.

7. Hoxha E, Harendza S, Pinnschmidt H, Panzer U, Stahl RA. PLA2R antibody levels and clinical outcome in patients with membranous nephropathy and non-nephrotic range proteinuria under treatment with inhibitors of the renin-angiotensin system. PloS One. 2014;9(10):e110681.

8. Cravedi P, Abbate M, Gagliardini E, et al. Membranous nephropathy associated with IgG4-related disease. Am J Kidney Dis. 2011;58(2):272-275.

9. Yamaguchi M, Ando M, Yamamoto R, et al. Patient age and the prognosis of idiopathic membranous nephropathy. PLoS One. 2014;9(10):e110376.

10. Hoxha E, Harendza S, Pinnschmidt H, Panzer U, Stahl RA. M-type phospholipase $\mathrm{A} 2$ receptor autoantibodies and renal function in patients with primary membranous nephropathy. Clin J Am Soc Nephrol. 2014;9(11):1883-1890.

11. Wang Y, Wang GP, Li BM, Chen QK. Clinicopathological analysis of idiopathic membranous nephropathy in young adults. Gen Mol Res. 2015;14(2):4541-4548.

12. Ronco P, Debiec H. Pathophysiological advances in membranous nephropathy: time for a shift in patient's care. Lancet. 2015;385(9981):1983-1992.

13. Ponticelli C, Passerini P. Management of idiopathic membranous nephropathy. Expert Opin Pharmacother. 2010;11(13):2163-2175.

14. Praga M, Rojas-Rivera J. Glomerular disease: Predicting outcomes in idiopathic membranous nephropathy. Nat Rev Nephrol. 2012;8(9):496-498.

15. Churg J, Ehrenreich T. Membranous nephropathy. Perspect Nephrol Hypertens. 1973;1 Pt 1:443-448.
16. Ponticelli C, Glassock RJ. Glomerular diseases: membranous nephropathy--a modern view. Clin J Am Soc Nephrol. 2014;9(3):609-616.

17. Hartono C, Chung M, Kuo SF, Seshan SV, Muthukumar T. Bortezomib therapy for nephrotic syndrome due to idiopathic membranous nephropathy. J Nephrol. 2014;27(1):103-106.

18. Tomas NM, Beck LH Jr, Meyer-Schwesinger C, et al. Thrombospondin type-1 domain-containing 7A in idiopathic membranous nephropathy. N Engl J Med. 2014;371(24):2277-2287.

19. Tran TH, J Hughes G, Greenfeld C, Pham JT. Overview of current and alternative therapies for idiopathic membranous nephropathy. Pharmacotherapy. 2015;35(4):396-411.

20. Hofstra JM, Fervenza FC, Wetzels JF. Treatment of idiopathic membranous nephropathy. Nat Rev Nephrol. 2013;9(8):443-458.

21. Chen Y, Schieppati A, Chen X, et al. Immunosuppressive treatment for idiopathic membranous nephropathy in adults with nephrotic syndrome. Cochrane Database Syst Rev. 2014;(10):CD004293.

22. Grupper A, Cornell LD, Fervenza FC, Beck LH Jr, Lorenz E, Cosio FG. Recurrent membranous nephropathy after kidney transplantation: treatment and long-term implications. Transplantation. Epub 2015 Dec 30 .

23. Yamamoto S, Inaba S, Yoshida R, et al. Clinicopathological characteristics of the focal and segmental form of idiopathic membranous nephropathy: comparison with the typical form of this disease. Acta Paediatr Jpn. 1997;39(3):349-353.

24. Chen X, Chen Y, Shi K, et al. Comparison of prognostic, clinical, and renal histopathological characteristics of overlapping idiopathic membranous nephropathy and IgA nephropathy versus idiopathic membranous nephropathy. Sci Rep. 2017;7(1):11468.

25. Shiiki H, Saito T, Nishitani Y, et al. Prognosis and risk factors for idiopathic membranous nephropathy with nephrotic syndrome in Japan. Kidney Int. 2004;65(4):1400-1407.

26. Gandolfo MT, Verzola D, Salvatore F, et al. Gender and the progression of chronic renal diseases: does apoptosis make the difference? Minerva Urol Nefrol. 2004;56(1):1-14.

27. Rood IM, Merchant ML, Wilkey DW, et al. Increased expression of lysosome membrane protein 2 in glomeruli of patients with idiopathic membranous nephropathy. Proteomics. 2015; 15(21):3722-3730.

28. Debiec H, Ronco P. Immunopathogenesis of membranous nephropathy: an update. Semin Immunopathol. 2014;36(4):381-397.

29. Tan Y, Yang D, Fan J, Chen Y. Elevated levels of immunoglobulin E may indicate steroid resistance or relapse in adult primary nephrotic syndrome, especially in minimal change nephrotic syndrome. J Int Med Res. 2011;39(6):2307-2313.

30. Tain YL, Chen TY, Yang KD. Implication of serum IgE in childhood nephrotic syndrome. Pediatr Nephrol. 2003;18(12):1211-1215.

31. Beck LH Jr, Bonegio RG, Lambeau G, et al. M-type phospholipase A2 receptor as target antigen in idiopathic membranous nephropathy. N Engl J Med. 2009;361(1):11-21.

32. Zhang Q, Huang B, Liu X, et al. Ultrasensitive quantitation of anti-phospholipase A2 receptor antibody as a diagnostic and prognostic indicator of idiopathic membranous nephropathy. Sci Rep. 2017;7(1):12049.

33. Xu NX, Xie QH, Sun ZX, et al. Renal phospholipase A2 receptor and the clinical features of idiopathic membranous nephropathy. Chin Med $J$ (Engl). 2017;130(8):892-898.

34. Wu X, Wen S, Zhu X, et al. [Diagnostic value of renal phospholipase A2 receptor and serum anti-phospholipase A2 receptor antibody in membranous nephropathy]. Zhong Nan Da Хие Xие Bao Yi Xиe Ban. 2017;42(4):395-399. Chinese. 
The International Journal of Nephrology and Renovascular Disease is an international, peer-reviewed open access journal focusing on the pathophysiology of the kidney and vascular supply. Epidemiology, screening, diagnosis, and treatment interventions are covered as well as basic science, biochemical and immunological studies. The manuscript management system is completely online and includes a very quick and fair peer-review system, which is all easy to use. Visit http://www. dovepress.com/testimonials.php to read real quotes from published authors.

Submit your manuscript here: https://www.dovepress.com/international-journal-of-nephrology-and-renovascular-disease-journal 